\title{
A Novel Optical Decision Circuit Based on a Mach-Zehnder or Michelson Interferometer and Gain-Clamped Semiconductor Optical Amplifiers
}

\author{
Geert Morthier, Member, IEEE, Jinying Sun, Tim Gyselings, and Roel Baets, Member, IEEE
}

\begin{abstract}
A novel optical decision circuit based on a MachZehnder or Michelson interferometer with a gain-clamped semiconductor optical amplifier in each arm is proposed. Simulation results show that the component, of which the decision threshold can easily be modified through adjustment of the currents in both amplifiers, exhibits excellent reshaping capacities.
\end{abstract}

Index Terms - Optical communication, optical pulse shaping, semiconductor optical amplifiers.

\section{INTRODUCTION}

D UE TO THE ACCUMULATION of noise, crosstalk, or distortion in long-haul optical communication systems, regeneration of the signals at intermediate distances is required in order to guarantee low bit-rate operation. In its simplest form, this regeneration is reduced to an amplification of the signals (so-called 1R regeneration) e.g., using EDFA's. However, the advanced, high-bit-rate communication systems of the future will also require reshaping ( $2 R$ regeneration) and retiming (3R regeneration) of the individual bits in a datastream. For the sake of simplicity, cost and speed, this regeneration is preferrably done in the optical domain.

An essential component for the optical $2 \mathrm{R}$ and $3 \mathrm{R}$ regeneration is the optical decision circuit, in which all signal levels below a certain decision threshold are transformed into a constant low level and all signal levels above the decision threshold are transformed into a constant high level. In recent years, several such optical decision circuits have been proposed. In [1], regeneration of return-to-zero (RZ) pulses using a nonlinear fiber-loop mirror and clock extraction with a mode-locked laser is proposed. The necessity of fiber components makes the circuit rather spacious and unsuitable for integration however. A more compact solution is provided by $Q$-switched distributed-feedback (DFB) lasers [2] or sidemode injection-locked DFB lasers [3], but the reliability of both solutions is yet to be demonstrated. In fact, to our knowledge an optical decision circuit that is bit rate and modulation format transparant, that is suitable for integration, and that operates on the signal itself instead of on a pump or external pulse sequence, and with a truly digital (i.e., nonperiodic) characteristic has not yet been reported.

Manuscript received February 10, 1998; revised April 2, 1998. This work was supported by the European ACTS Project REPEAT (AC-305).

The authors are with the Department of Information Technology, University of Gent-IMEC, B-9000 Gent, Belgium.

Publisher Item Identifier S 1041-1135(98)05349-X.

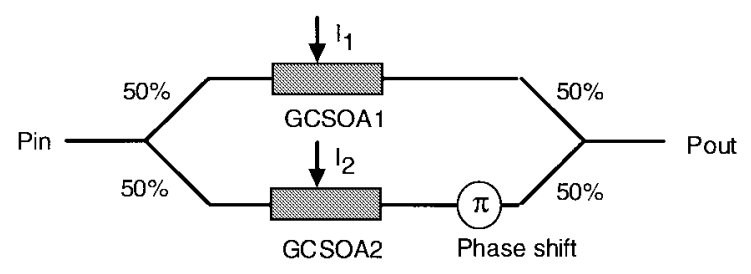

(a)

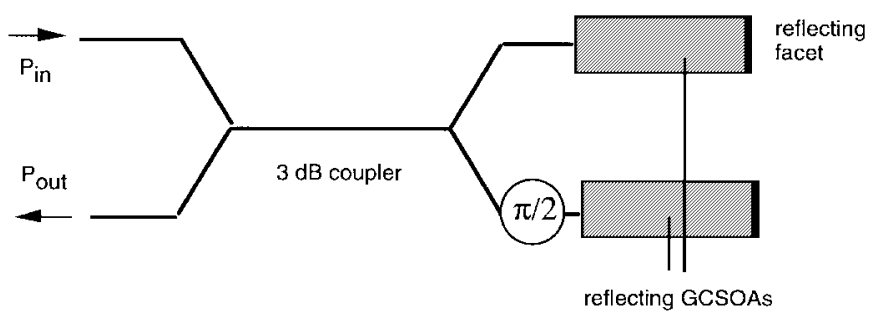

(b)

Fig. 1. (a) Symmetrical MZI-based implementation and (b) symmetrical MI-based implementation of an optical decision circuit.

In this letter, we present an optical decision circuit [4], based on Mach-Zehnder or Michelson interferometers (MZI or MI) and gain-clamped semiconductor optical amplifiers (GCSOA's) [5], [6], which does possess all these properties. In the next section, we start with the description of the decision circuits and we explain their principle of operation. In section III, we present simulation results of the decision circuit, obtained using a longitudinal model for the semiconductor optical amplifiers (SOA's), and we give preliminary dynamic characteristics. Conclusions will be drawn in Section IV.

\section{BASIC STRUCTURES}

A possible structure of the MZI-based circuit, with symmetric splitters and identical GCSOA's in both arms, is shown in Fig. 1(a). The GCSOA's need to be traveling-wave amplifiers in this case and can be implemented as DFB or distributed Bragg reflector (DBR) lasers with antireflection (AR)-coating on both facets. Other implementations of this MZI-based circuit, e.g., with asymmetric splitters and different GCSOA's in both arms, can also be used and give more freedom in the design, but the symmetric structure will be discussed here to illustrate the principle. The phase shift of $\pi$ can be implemented as a phase modulator or as a path length difference. The two GCSOA's are assumed to be completely identical 


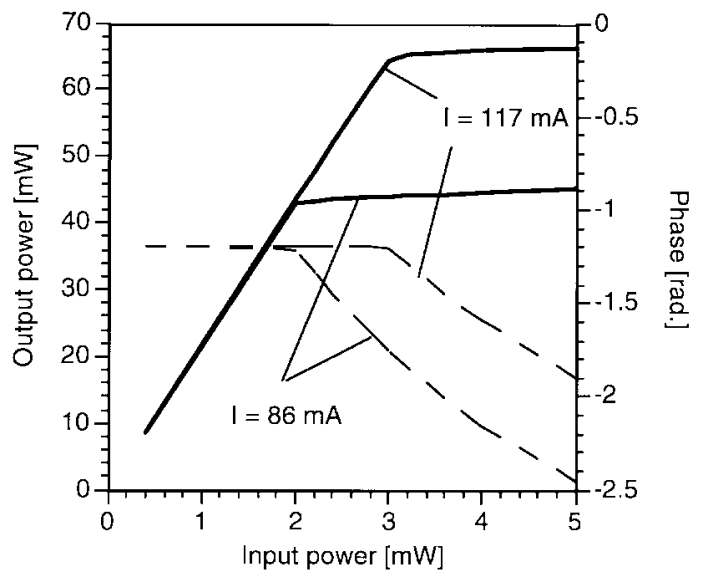

Fig. 2. Amplitude (-) and phase (- - -) characteristics of two GCSOA's with different bias currents (86 and $117 \mathrm{~mA}$ ).

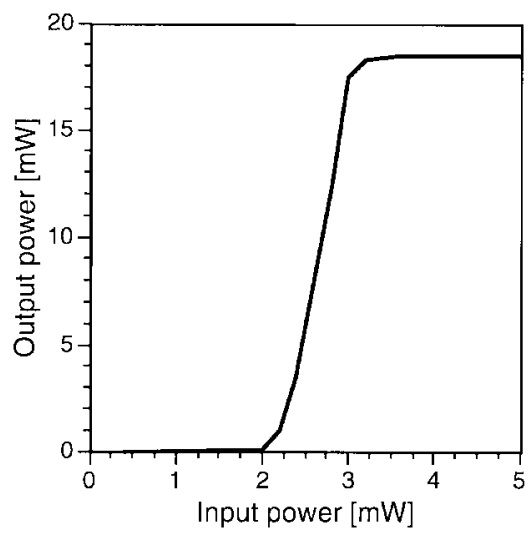

Fig. 3. Output power versus input power of a symmetric decision circuit.

and to have a different bias current. They, therefore, give an identical and constant amplification and phase shift below the saturation power, but they exhibit a different saturation power. Calculated characteristics of 2 GCSOA's with different bias current are shown in Fig. 2. For equal input fields (as is the case in both arms of the interferometer for symmetric splitters), both amplifiers obviously give equal output fields in the linear regime. Hence, the phase shift of $\pi$ in one of the arms causes total destructive interference between both output fields resulting in a zero output of the interferometer. As can also be seen in Fig. 3, above the saturation input power of both amplifiers, the output power of both amplifiers is constant but different. Furthermore, due to additional phase shifts accompanying the saturation in each GCSOA, the phase difference between both interference arms becomes different from $\pi$. Due to this difference in output power in both arms and the phase difference different from $\pi$, there is no longer total destructive interference. However, the fact that the amplifier output powers in both arms and the phase difference between both arms remain constant as the input power varies above the saturation powers causes the output power of the interferometer to be constant as well. In between the saturation power of the first amplifier and the saturation power of a second amplifier, there is a gradual increase in the output power of the interferometer.

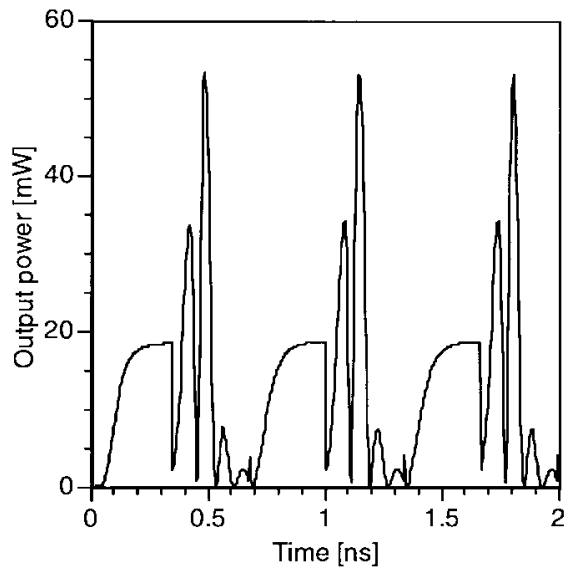

Fig. 4. Time evolution of the output power of the symmetric decision circuit for a periodic $3-\mathrm{Gb} / \mathrm{s}$ sequence of spaces and marks.

An alternative for the MZI-based circuit is the MI-based circuit depicted in Fig. 1(b). In this case we again assume that the directional coupler gives 50/50 coupling and that both GCSOA's are identical, but other implementations with asymmetric couplers and different GCSOA's in both arms are again possible. Important, however, is that in this configuration the amplifiers must be reflecting. They can, therefore, be implemented as DFB lasers with one AR-coated facet and one cleaved or HR-coated facet. As in the MZI-based structure, both amplifiers are assumed to have a different bias current such that they exhibit equal amplification and phase shift below the saturation power and a different saturation power. The operation principle of this MI-based decision circuit is then identical to that of the MZI-based decision circuit. It must be emphasized though that an identical amplification for different saturation powers can only be obtained with GCSOA's and not with ordinary traveling-wave amplifiers.

\section{Simulation Results ON THE MZI}

In this section, we consider a MZI-based decision circuit with a symmetric interferometer and traveling-wave GCSOA's, consisting of an amplifying section surrounded by passive DBR sections on either side. The parameters used in the simulation of the GCSOA's are summarized in Table I. The material parameters (i.e., $C_{1}-C_{6}$ ) are based on the values used in [7], the other parameters in Table I were chosen with the intent of obtaining about $20-\mathrm{dB}$ amplifier gain. No gain suppression (or explicit power dependence of the gain g) has been taken into account. The simulations have been performed using the longitudinal computer model CLADISS [7]. The GCSOA's exhibit lasing with a threshold current of $24.8 \mathrm{~mA}$ and at an emission wavelength of $1.5298 \mu \mathrm{m}$. The wavelength of the injected signal was chosen to be $1.55 \mu \mathrm{m}$, i.e., sufficiently far from the lasing wavelength and the Bragg wavelength of the Bragg sections. It can further be noticed that the (monomolecular, bimolecular and Auger) recombination parameters mainly affect the threshold current of the GCSOA's, and hence also the currents corresponding with a certain saturation power, and the damping of the relaxation oscillations during the switching. 
TABLE I

GCSOA Parameters Used in the Numerical Simulations

\begin{tabular}{|c|c|c|}
\hline Parameter & Value & Unit \\
\hline Length of the Bragg sections & 400 & $\mu \mathrm{m}$ \\
\hline Period of the Bragg gratings & 233 & nm \\
\hline Coupling coefficient of the Bragg gratings & 7.5 & $\mathrm{~cm}^{-1}$ \\
\hline Effective index of the Bragg sections & 3.283 & \\
\hline Length of the active section & 1000 & $\mu \mathrm{m}$ \\
\hline Cross section of the waveguide & 0.3 & $\mu \mathrm{m}^{2}$ \\
\hline Confinement factor & 0.4 & \\
\hline Internal loss & 25 & $\mathrm{~cm}^{-1}$ \\
\hline Monomolecular recombination coefficient & $0.16710^{9}$ & $s^{-1}$ \\
\hline Bimolecular recombination coefficient & $10^{-16}$ & $\mathrm{~cm}^{3} / \mathrm{s}$ \\
\hline Auger recombination coefficient & $310^{-23}$ & $\mathrm{~cm}^{6} / \mathrm{s}$ \\
\hline Effective index without injection & 3.283 & \\
\hline Change of $n_{\text {eff }}$ with carrier density & $-1.6910^{-20}$ & $\mathrm{~cm}^{3}$ \\
\hline \multicolumn{3}{|l|}{ Gain $g=C_{1}\left(E-C_{2}\right)^{6} N-C_{1}\left(E-C_{5}\right)^{c 6}$} \\
\hline $\mathrm{C}$, & $4.6810^{15}$ & $\mathrm{~cm}^{2}$ \\
\hline $\mathrm{C}_{\text {I }}$ & 0.7477 & $\mathrm{eV}$ \\
\hline $\mathrm{C}_{3}$ & 0.7743 & \\
\hline $\mathrm{C}_{1}$ & $77.1610^{4}$ & $\mathrm{~cm}^{-1}$ \\
\hline $\mathrm{C}_{3}$ & 0.6237 & $\mathrm{eV}$ \\
\hline $\mathrm{C}_{\mathrm{G}}$ & 4.1727 & \\
\hline
\end{tabular}

Fig. 2 shows the amplification and phase shift characteristics of the GCSOA's under different bias currents (86 and 117 $\mathrm{mA})$. The output power versus input power characteristic of the circuit of Fig. 1(a), calculated using the characteristics of Fig. 2, is shown in Fig. 3. As can be seen, the output power is fairly constant below $2 \mathrm{~mW}$ and above $3 \mathrm{~mW}$. The additional phase shift due to saturation of the GCSOA with lowest bias is only $0.5 \mathrm{rad}$ here. Ideally, this phase shift should be $\pi$, but this value is not easily obtained with realistic values for material and device parameters [4].

To get a first impression of the speed limits, we have performed time-domain simulations with a $3-\mathrm{Gb} / \mathrm{s}$ periodic sequence of rectangular pulses as input. The power level of the marks has been chosen as $4 \mathrm{~mW}$, while the power levels of the spaces have been chosen as $0.4 \mathrm{~mW}$. Fig. 4 shows the temporal variation of the output power. Very strong ringing can be remarked during the switching-off of the decision circuit. The lasing in both GCSOA's switches on during this ringing and is accompanied by relaxation oscillations. The strong ringing in Fig. 4 is caused more exactly by the fact that the relaxation oscillations in both GCSOA's exhibit a different frequency and start after different delay times, which gives rise to very large phase differences between both arms. This ringing seems to limit the application of the decision circuit, at least with the specific GCSOA parameters of Table I, to not much more than
$3 \mathrm{~Gb} / \mathrm{s}$. However, it is believed that after optimization of the parameters of the GCSOA, for example, aimed at increasing the damping of the relaxation oscillations and at decreasing the linewidth enhancement factor [8], an operation at much higher bit rates might be feasible. The difference in switch-on delay between both arms can be decreased by implementing a delay (necessary to get a phase difference of $(2 k+1) \pi$ anyway) in the arm with the highest bias.

\section{CONCLUSION}

We have proposed new structures allowing all-optical $2 \mathrm{R}$ signal regeneration and showing potential for implementation as photonic integrated circuits. Numerical simulations of the structures have been performed and have shown that nearly ideal optical decision characteristics result. First simulations furthermore show that the chirp introduced by the device is of the same order of magnitude as the chirp introduced by MZI all-optical wavelength converter.

The operation of the new decision circuit has been illustrated for the theoretical case where two completely identical amplifiers are available, which may seem unrealistic. However, this case can easily be obtained in practice by adding a variable (or trimmable) attenuation and a variable (or trimmable) phase shift in one arm such that the two arms of the interferometer give equal net gains and a net phase difference of $\pi$ in the linear regime. In addition, a circuit built with two nearly identical GCSOA's is believed to give a nice decision characteristic with a small but finite output for small input powers.

\section{REFERENCES}

[1] J. K. Lucek and K. Smith, "All-optical signal regenerator," Opt. Lett., vol. 18 , pp. 1226-1228, 1993.

[2] B. Sartorius and M. Mohrle, "Mirror modulated lasers: a concept for high speed transmitters," Electron. Lett., vol. 32, pp. 1781-1782, 1996.

[3] K. Inoue and M. Yoshimo, "Bistability and waveform reshaping in a DFB-LD with side-mode injection," IEEE Photon. Technol. Lett., vol. 7, pp. 164-166, 1995.

[4] G. Morthier and R. Baets, patent application.

[5] P. Doussière, A. Jourdan, G. Soulage, P. Garabedian, C. Graverm, T. Fillion, E. Derouin, and D. Leclerc, "Clamped gain travelling wave semiconductor optical amplifier for wavelength division multiplexing applications," in Proc. Int. Semiconductor Laser Conf., Maui, HI, 1994 pp. $185-186$.

[6] L. Tiemeijer, P. J. A. Thijs, T. v. Dongen, J. J. M. Binsma, E. J. Jansen, and H. R. J. R. van Helleputte, "Reduced intermodulation distortion in $1300 \mathrm{~nm}$ gain-clamped MQW laser amplifiers," IEEE Photon. Technol. Lett., vol. 7, pp. 284-286, 1995.

[7] P. Vankwikelberge, G. Morthier, and R. Baets, "CLADISS—A longitudinal, multi-mode model for the analysis of the static, dynamic and stochastic behavior of diode lasers with distributed feedback," IEEE J. Quantum Electron., vol. 26, pp. 1728-1741, 1990.

[8] G. Morthier and P. Vankwikelberge, Handbook of Distributed Feedback Laser Diodes. Boston, MA: Artech House, 1997. 